\title{
Article \\ Theoretical Investigation of Subluminal Particles Endowed with Imaginary Mass
}

\author{
Luca Nanni
}

check for

updates

Citation: Nanni, L. Theoretical Investigation of Subluminal Particles Endowed with Imaginary Mass. Particles 2021, 4, 325-332. https: / / doi.org/10.3390/particles4020027

Academic Editor: Kazuharu Bamba

Received: 29 May 2021

Accepted: 15 June 2021

Published: 18 June 2021

Publisher's Note: MDPI stays neutral with regard to jurisdictional claims in published maps and institutional affiliations.

Copyright: (C) 2021 by the author. Licensee MDPI, Basel, Switzerland. This article is an open access article distributed under the terms and conditions of the Creative Commons Attribution (CC BY) license (https:// creativecommons.org/licenses/by/ $4.0 /)$.
Faculty of Natural Science, University of Ferrara, 44122 Ferrara, Italy; luca.nanni@edu.unife.it

\begin{abstract}
In this article, the general solution of the tachyonic Klein-Gordon equation is obtained as a Fourier integral performed on a suitable path in the complex $\omega$-plane. In particular, it is proved that this solution does not contain any superluminal components under the given boundary conditions. On the basis of this result, we infer that all possible spacelike wave equations describe the dynamics of subluminal particles endowed with imaginary mass. This result is validated for the Chodos equation, used to describe the hypothetical superluminal behaviour of the neutrino. In this specific framework, it is proved that the wave packet propagates in spacetime with subluminal group velocities and that it behaves as a localized wave for sufficiently small energies.
\end{abstract}

Keywords: tachyons; Klein-Gordon equation; tachyon-like Dirac equation; relativistic wave packet

PACS: 03.65.Pm; 03.65.Ge; 03.65.Db

\section{Introduction}

The physics of tachyons is an intriguing and fascinating subject that has attracted the attention of physicists in both the pre-relativistic [1-3] and post-relativistic epochs [4-10]. However, the majority of the scientific community still considers it a speculative theory, and there are only a few efforts aimed at finding the experimental evidence that could make it a candidate for extending the current Standard Model [11]. A comprehensive overview of the most relevant experiments dealing with the superluminal behaviour of waves and particles is detailed in [9,12-15]. If considered in the framework of quantum mechanics, tachyons exhibit completely unexpected behaviours [16-18]. In particular, half-integer free fermions endowed with imaginary mass behave like wave trains propagating with subluminal group velocities. The aim of this study is to verify whether this result can be generalized for any particle regardless of its spin. The basic idea is that the individual components of spinor wave functions, which are solutions of the tachyonic wave equations, must satisfy the tachyonic Klein-Gordon (TKG) equation as well [19]. Finding a general solution of the TKG equation without spacelike components means proving that any solution of the wave equations of particles with negative mass squared represents a wave packet that propagates with subluminal group velocity. As proposed by Salesi, these particles are called pseudotachyons (PTs), namely particles travelling with slower-than-light velocity but fulfilling the tachyonic energy-momentum relation $E^{2}=p^{2} c^{2}+\mu^{2} c^{4}$, where $\mu=i m$ denotes the imaginary mass. The obtained result will be validated for the Chodos equation [20] which describes the behaviour of a $\frac{1}{2}$-spin tachyon using a more different analytical approach than that discussed in [18].

\section{Preliminary Notions on the Fourier Transforms in the Complex Plane}

To facilitate the reading of Section 3 of this article, a brief review on the theory of the Fourier transform in the complex plane is presented [21]. 
Let $f(x)$ be an integrable function; its Fourier transform is defined as:

$$
\mathcal{F}[f(x)]=\widetilde{f}(\omega)=\int_{-\infty}^{\infty} f(x) e^{i \omega x} d x,
$$

while the inverse Fourier transform is:

$$
\mathcal{F}^{-1}[\widetilde{f}(\omega)]=\frac{1}{2 \pi} \int_{-\infty}^{\infty} \widetilde{f}(\omega) e^{-i \omega x} d \omega .
$$

Definitions (1) and (2) can be generalized by allowing $\omega$ to take complex values. To this end, let us suppose that $f(x)$ grows at most exponentially at infinity, i.e., $f(x)=\mathcal{O}\left(e^{c|x|}\right)$ as $x \rightarrow \pm \infty$ and $c>0$. Moreover, we split up $f(x)$ as follows:

$$
f(x)=f_{+}(x)+f_{-}(x): f_{+}(x)=0 \quad \forall x<0 \text { and } f_{-}(x)=0 \forall x>0
$$

The Fourier transform of the component $f_{+}(x)$ is:

$$
\tilde{f}_{+}(\omega)=\int_{0}^{\infty} f_{+}(x) e^{i R e(\omega) x} e^{-\operatorname{Im}(\omega) x} d x
$$

It is evident that:

$$
\left|\widetilde{f}_{+}(\omega)\right| \leq \int_{0}^{\infty}\left|f_{+}(x)\right| e^{-\operatorname{Im}(\omega) x} d x,
$$

and the integral converges provided $\operatorname{Im}(\omega)>c$. Therefore, $\widetilde{f}_{+}(\omega)$ exists and is holomorphic for $\operatorname{Im}(\omega)>c$.

Next, we need to extend the Fourier inversion theorem to recover $f_{+}(x)$ from $\widetilde{f}_{+}(\omega)$. To this end, let $F_{+}(x)=f_{+}(x) e^{-\alpha x}$, where $\alpha>c$, so that $\left[\widetilde{F}_{+}(\omega)\right]=\widetilde{f}_{+}(\omega+i \alpha)$ exists and is holomorphic for $\operatorname{Im}(\omega)>c-\alpha$, in particular for $\omega \in \mathbb{R}$, since $\alpha>c$. Thus, we can apply the Fourier transform inversion theorem obtaining:

$$
\begin{gathered}
F_{+}(x)=\frac{1}{2 \pi} \int_{-\infty}^{\infty} \widetilde{F}_{+}(\omega) e^{-i \omega x} d \omega \Rightarrow f_{+}(x) e^{-\alpha x}=\frac{1}{2 \pi} \int_{-\infty}^{\infty} \widetilde{f}_{+}(\omega+i \alpha) e^{-i \omega x} d \omega \\
\Rightarrow f_{+}(x)=\frac{1}{2 \pi} \int_{-\infty}^{\infty} \widetilde{f}_{+}(\omega+i \alpha) e^{-i(\omega+i \alpha) x} d \omega .
\end{gathered}
$$

The final integral corresponds to an integration along a horizontal contour in the complex $\omega$-plane:

$$
f_{+}(x)=\frac{1}{2 \pi} \int_{-\infty+i \alpha}^{\infty+i \alpha} \widetilde{f}_{+}(\omega) e^{-i \omega x} d \omega .
$$

Suppose $\tilde{f}_{+}(\omega)$ can be continued below $\operatorname{Im}(\omega)=c$, so that it is holomorphic in some region $\Omega_{+} \supset\{\omega: \operatorname{Im}(\omega)>c\}$ except for singularities at $\omega=a_{1}, a_{2}, \cdots$. By the deformation theory, the inversion contour $\Gamma_{+}=\{x+i \alpha:-\infty<x<\infty\}$ may be deformed into $\Omega_{+}$provided it passes above the singularities of $\widetilde{f}_{+}(\omega)$. Since the singularities of $\widetilde{f}_{+}(\omega)$ are below the inversion contour, for $x<0$, we can close the contour at $+i \infty$. This gives the expected result that $f_{+}(x)=0$ for $x<0$. For $x>0$, we would need to close the contour in $\operatorname{Im}(\omega)<0$, picking up the contributions from the singularities in $\widetilde{f}_{+}(\omega)$ and giving a nonzero value of $f_{+}(x)$.

The same procedure works for $f_{-}(x)$ with everything upside down, namely $\widetilde{f}_{-}(\omega)$ can be continued above $\operatorname{Im}(\omega)=-c$, so that it is holomorphic in some region $\Omega_{-} \supset$ 
$\{\omega: \operatorname{Im}(\omega)<-c\}$ except for singularities at $\omega=b_{1}, b_{2}, \cdots$. If there is a non-empty overlap region $\Omega=\Omega_{+} \cap \Omega_{-} \backslash\left\{a_{i}\right\} \cup\left\{b_{j}\right\}$, where $\left\{a_{i}\right\}$ are the singularities of $\widetilde{f}_{+}(\omega)$ and $\left\{b_{j}\right\}$ are the singularities of $\tilde{f}_{-}(\omega)$, then the Fourier transform of $f(x)$ is defined as:

$$
\widetilde{f}(\omega)=\widetilde{f}_{+}(\omega)+\widetilde{f}_{-}(\omega),
$$

for $\omega \in \Omega$. Moreover, if $\Gamma_{+}$and $\Gamma_{-}$can be deformed into the same contour $\Gamma \subset \Omega$, with $\Gamma$ above the singularities of $\widetilde{f}_{+}(\omega)$ and below the singularities of $\widetilde{f}_{-}(\omega)$, then:

$$
f(x)=\frac{1}{2 \pi} \int_{\Gamma} \tilde{f}(\omega) e^{-i \omega x} d \omega .
$$

What is discussed in this section represents the method that will be implemented below to solve the TKG equation.

\section{Dynamics of Wave Packets in the TKG Equation}

The TKG equation can be obtained by quantizing the tachyonic energy-momentum relation using the operators $E \rightarrow i \hbar \partial / \partial \mathrm{t}$ and $p \rightarrow-i \hbar c \nabla$ :

$$
\left(\hbar^{2} \frac{\partial^{2}}{\partial t^{2}}-\hbar^{2} c^{2} \nabla^{2}-m^{2} c^{4}\right) \psi(\boldsymbol{r}, t)=0 .
$$

For free scalar particles, Equation (10) can be solved by Fourier transform in time, which allows decomposing the wave function $\psi(\boldsymbol{r}, t)$ in its monochromatic temporal components, using the initial conditions given for $\left.\psi(r, t)\right|_{r=0}$ and $\partial \psi(r, t) /\left.\partial r\right|_{r=0}$.

To simplify the following discussion, we suppose that the motion of the particle takes place along the $z$-axis. The solution is a wave packet represented by the following inverse Fourier transform:

$$
\psi(z, t)=\frac{1}{2 \pi} \int_{\Gamma}\left[A(\omega) e^{i K(\omega) z}+B(\omega) e^{-i K(\omega) z}\right] e^{-i \omega t} d \omega,
$$

where $\Gamma$ is a path in the $\omega$-plane. The quantity $\omega$ is defined as $\omega= \pm E / \hbar$, and, for a tachyon, it is always a real quantity except when $E= \pm \mu c^{2}$ (this last statement will be clarified in Section 4 where we calculate the group velocity of the tachyonic wave packet). In fact:

$$
E= \pm \gamma \mu c^{2}= \pm\left(1-\frac{u^{2}}{c^{2}}\right)^{-1 / 2} i m c^{2}= \pm\left(\frac{u^{2}}{c^{2}}-1\right)^{-1 / 2} m c^{2},
$$

where $\gamma$ is the tachyonic Lorentz factor and $u>c$ is the classical tachyonic velocity. Returning to Equation (11), the term $K(\omega)$ is the dispersion relation whose characteristic equation is:

$$
K^{2}(\omega)=\frac{\omega^{2}-\omega_{0}^{2}}{c^{2}} \text { where } \omega_{0}^{2}=-m^{2} c^{4} / \hbar^{2} .
$$

Equation (13) is obtained from Equation (10) upon factorisation $\psi(z, t)=\varphi(z) e^{-i \omega t}$. The aim is to prove that Equation (1) admits a general solution in which no spacelike components arise. In other words, we want to prove that this solution is completely analogous to the one that solves the ordinary KG equation, where $\omega$ is always real, and tends to the solution of the d'Alembert wave equation as the mass energy tends to zero. We point out that the spacelike components are those associated with complex values of $\omega$, and therefore of $K(\omega)$.

The coefficients $A(\omega)$ and $B(\omega)$ are calculated once the following boundary conditions have been set [21]:

$$
f(t)=\psi(0, t) \text { and } f^{\prime}(t)=\partial \psi(z, t) /\left.\partial z\right|_{z=0} .
$$


As we will see, these conditions determine the choice of the path $\Gamma$ of integration. Using the boundary conditions in Equation (14), we obtain [21]:

$$
\left\{\begin{array}{l}
A(\omega)=\frac{1}{2} \int_{-\infty}^{\infty}\left[f(t)-i \frac{f^{\prime}(t)}{K(\omega)}\right] e^{i \omega t} d t \\
B(\omega)=\frac{1}{2} \int_{-\infty}^{\infty}\left[f(t)+i \frac{f^{\prime}(t)}{K(\omega)}\right] e^{i \omega t} d t
\end{array}\right.
$$

The computation of the integrals in Equation (15) can be simplified if we consider $f(t)$ as a semi-infinite wave train with a sharp edge. In other words, we can set a time $t_{0}$ such that $\forall t<t_{0} \Rightarrow f(t)=0$. This is equivalent to state that, at the time $t_{0}$, the wave is created. However, in order to ensure the convergence of the integral in Equation (11) as $t \rightarrow t_{0^{+}}$, the function $f(t)$ tends to zero. From now on, we assume $t_{0}=0$. Under this assumption and $f(t)$ and $f^{\prime}(t)$ being infinite wave trains with a sharp edge, it is necessary that $\omega$ runs on a path equivalent to the line $(-\infty+i \alpha, \infty+i \alpha)$ where $\alpha>0$, in order to obtain the convergence of the integrals in Equation (15).

Let us now set the following further condition:

$$
B(\omega)=0 \Rightarrow f^{\prime}(t)=\frac{1}{2 \pi} i \int_{\Gamma} K(\omega) \mathcal{F}(f) e^{-i \omega t} d \omega,
$$

where $\mathcal{F}(f)$ is the Fourier transform of the function $f(t)$. Using Equation (16), the first of the integrals in Equation (15) becomes:

$$
A(\omega)=\int_{-\infty}^{\infty} f(t) e^{i \omega t} d t=\mathcal{F}(f) .
$$

Substituting the integral in Equation (17) into Equation (11), we obtain:

$$
\psi(z, t)=\frac{1}{4 \pi} \int_{\Gamma} \mathcal{F}(f) e^{i K(\omega) z-i \omega t} d \omega .
$$

Since $f(t)$ is a semi-infinite sharp-edged wave train, it can be written explicitly as:

$$
f(t)=\theta(t) e^{-\left.i \omega\right|_{t_{0}} t}
$$

where $\theta(t)$ is the Heaviside function. The Fourier transform of the function given in Equation (19) is [22]:

$$
\mathcal{F}(f)=i \frac{1}{\omega-\left.\omega\right|_{t_{0}}} .
$$

Substituting Equation (20) into Equation (18), we obtain the explicit form of the kernel:

$$
\psi(z, t)=\frac{1}{2 \pi} \int_{\Gamma} \frac{i}{\omega-\left.\omega\right|_{t_{0}}} e^{i K(\omega) z-i \omega t} d \omega .
$$

To solve the integral in Equation (21), we need to find the integration path $\Gamma$. In order to satisfy the initial conditions at $z=0$, all singularities of the Fourier coefficient $A(\omega)$ must lie under the path $\Gamma$ (as discussed in Section 2), so that the integral in Equation (11) vanishes as $t<t_{0}$, after closing $\Gamma$ in the upper half-plane. This is the mathematical representation of the fact that, since the wave function is a semi-infinite train with a sharp edge, the integrals in Equation (15) give rise to singularities on the real axis of the complex plane, except for the two branch points at $\omega= \pm i \omega_{0}$. Only non-physical expressions for $f(t)$ and $f^{\prime}(t)$ (namely, not suitable for a wave function) can produce singularities in the upper half-plane. The two branch points come from the dispersion relation:

$$
K(\omega)= \pm \frac{1}{c}\left(\omega^{2}+\omega_{0}^{2}\right)^{1 / 2}= \pm \frac{1}{c}\left[\left(\omega+\left|\omega_{0}\right|\right)\left(\omega-\left|\omega_{0}\right|\right)\right]^{1 / 2} .
$$


There are two possible kinds of cuts: the segment $\left(-i \omega_{0}, i \omega_{0}\right)$ or the pair of halflines $\left(i \omega_{0}, i \omega_{0}+\infty\right)$ and $\left(-i \omega_{0},-i \omega_{0}+\infty\right)$. Since the path $\Gamma$ must lies above the real axis, we have to study how it behaves when it meets the cut. It could go around the cut or run parallel to the real axis both of these choices are mathematically correct. However, the second choice would lead to superluminal components given by the upper cut, and the group velocity associated with the wave function will be greater than the speed of light. The criterion for discriminating the choice of the integration path is that, at the limit $\left|\omega_{0}\right| \rightarrow 0$, the dispersion relation $K(\omega)$ tends to $\omega / c$ which represents the typical dispersion relation of a wave packet that satisfies the ordinary wave equation, which does not admit tachyonic components. This condition excludes the cuts given by the pair of half-lines $\left(i \omega_{0}, i \omega_{0}+\infty\right)$ and $\left(-i \omega_{0},-i \omega_{0}+\infty\right)$ and leads to the correct dispersion relation $K(\omega)=\left|\omega^{2}+\omega_{0}^{2}\right|^{1 / 2} / c$ for $\omega>0$ and $K(\omega)=-\left|\omega^{2}+\omega_{0}^{2}\right|^{1 / 2} / c$ for $\omega<0$.

The wave function in Equation (21) has a completely general form, and the integration path $\Gamma$ is uniquely determined by the choice of the initial conditions (Equations (14), (16) and (19)). Therefore, it is expected that, whatever the tachyonic equation considered, the wave packet describing the dynamics of the particle always propagates with subluminal velocity, even if it is associated with a negative mass squared.

\section{Validation of the Obtained Result for Half-Integer Spin Tachyon}

The results obtained in Section 3 should be validated for each tachyonic wave equation. However, to the best of our knowledge, the only tachyonic wave equation formulated for massive particles is that of Chodos which describes the motion of a particle with half-integer spin [23]. This equation is generally used to investigate neutrinos exhibiting superluminal behaviours [24]. Therefore, we work on this equation with the aim to obtain a solution in the form of a Gaussian wave packet from which we calculate the group velocity. To this purpose, we formulate the equation of the envelope function that characterises the wave packet.

The Chodos equation is obtained from the Tanaka Lagrangian [25]. For a tachyonic particle moving along the $z$-axis, this (Hermitian) Lagrangian reads:

$$
\mathcal{L}=i \hbar \bar{\psi} \gamma^{5} \gamma^{0} \partial_{t} \psi-i \hbar c \bar{\psi} \gamma^{5} \gamma^{3} \partial_{z} \psi-m c^{2} \bar{\psi} \psi,
$$

where $m=|\mu|, \bar{\psi}_{t}=\psi_{t}^{\dagger} \gamma^{0}$ and $\gamma^{5}=i \gamma^{0} \gamma^{1} \gamma^{2} \gamma^{3}$. Introducing the Lagrangian (Equation (23)) in the Euler-Lagrange equations, we obtain the Chodos equation:

$$
\left(i \hbar \gamma^{5} \gamma^{0} \partial_{t}-i \hbar c \gamma^{5} \gamma^{3} \partial_{z}-m c^{2}\right) \psi=0
$$

The spacelike property of Equation (24) is given by the operator $\gamma^{5}$ and not by the imaginary mass [25]. We seek solutions that have the form of a Gaussian wave packet:

$$
\psi^{ \pm}(z, t)=\mathcal{N}\left(\begin{array}{c}
\varphi_{1}^{ \pm} \\
\varphi_{2}^{ \pm}
\end{array}\right) f^{ \pm}(z, t) \exp \left\{ \pm i\left(k z-\omega^{ \pm} t\right)\right\},
$$

where \pm denotes the solutions with positive and negative frequencies, $\varphi_{1,2}^{ \pm}$are the spinor components, $f^{ \pm}(z, t)$ is the Gaussian envelope function and $\mathcal{N}$ is the normalization constant. Before formulating the equation for the function $f^{ \pm}(z, t)$, we need to calculate the spinor components for the particle and antiparticle states. To this purpose, we substitute the plane wave component of Equation (25) into Equation (24) and obtain a system of four algebraic equations from which the spinor components can be calculated:

$$
\left(\begin{array}{cccc}
i \hbar c \partial_{z}-m c^{2} & 0 & -i \hbar \partial_{t} & 0 \\
0 & -i \hbar c \partial_{z}-m c^{2} & 0 & -i \hbar \partial_{t} \\
i \hbar \partial_{t} & 0 & -i \hbar c \partial_{z}-m c^{2} & 0 \\
0 & i \hbar \partial_{t} & 0 & i \hbar c \partial_{z}-m c^{2}
\end{array}\right)\left(\begin{array}{c}
\varphi_{1}^{+} \\
\varphi_{2}^{+} \\
\varphi_{1}^{-} \\
\varphi_{2}^{-}
\end{array}\right) e^{ \pm i\left(k z-\omega^{ \pm} t\right)}=0 .
$$


Multiplying Equation (26) with its adjoint, we obtain four TKG equations. This equation can be easily solved by the Cramer rule and we obtain:

$$
\varphi_{1}^{+}=\varphi_{2}^{-}=-\left(\frac{\omega}{k c+\omega_{0}}\right)^{\frac{1}{2}} ; \varphi_{2}^{+}=\varphi_{1}^{-}=\left(\frac{\omega}{k c-\omega_{0}}\right)^{\frac{1}{2}} .
$$

where $\omega_{0}=m c^{2} / \hbar$. The spinor components of Equation (27) can be rewritten in parametric form. To this end, we set the classical tachyonic velocity as $u=\eta c$ with $\eta>1$. Then, Equation (27) becomes:

$$
\varphi_{1}^{+}=\varphi_{2}^{-}=-\left[\frac{\left(\eta^{2}-1\right)^{-\frac{1}{2}}}{\eta\left(\eta^{2}-1\right)^{-\frac{1}{2}}+1}\right]^{\frac{1}{2}} ; \varphi_{2}^{+}=\varphi_{1}^{-}=\left[\frac{\left(\eta^{2}-1\right)^{-\frac{1}{2}}}{\eta\left(\eta^{2}-1\right)^{-\frac{1}{2}}-1}\right]^{\frac{1}{2}} .
$$

We can now formulate the equation in $f^{ \pm}(z, t)$ which allows us to calculate both the group velocity of the wave packet and the explicit form of the envelope function. Substituting Equation (25) into Equation (24), we obtain:

$$
\left\{\begin{array}{c}
\left(\frac{\partial}{\partial t}-\left.c \frac{\varphi_{1}^{+}}{\varphi_{1}^{-}}\right|_{\eta_{0}} \frac{\partial}{\partial z}+\left.\frac{2 m c^{2}}{i \hbar} \frac{\varphi_{1}^{+}}{\varphi_{1}^{-}}\right|_{\eta_{0}}\right. \\
\left(\frac{\partial}{\partial t}+\left.c \frac{\varphi_{2}^{-}}{\varphi_{2}^{+}}\right|_{\eta_{0}} \frac{\partial}{\partial z}+\left.\frac{2 m c^{2}}{i \hbar} \frac{\varphi_{2}^{-}}{\varphi_{2}^{+}}\right|_{\eta_{0}}\right) f^{-}(z, t)=0
\end{array},\right.
$$

where $\eta_{0}$ is the centre of the Gaussian envelope function. The numerical coefficient of the second term in Equation (29) is just the group velocity of the wave packet [26]:

$$
v_{g}=-\left.c \frac{\varphi_{1}^{+}}{\varphi_{1}^{-}}\right|_{\eta_{0}}=\left.c \frac{\varphi_{2}^{-}}{\varphi_{2}^{+}}\right|_{\eta_{0}}=c\left[\frac{\eta_{0}\left(\eta_{0}^{2}-1\right)^{-1 / 2}-1}{\eta_{0}\left(\eta_{0}^{2}-1\right)^{-1 / 2}+1}\right]^{1 / 2}<c \forall \eta_{0}>c
$$

As expected, Equation (30) proves that the group velocity is always lower than the speed of light, confirming the result obtained in Section 3. From Equation (30), we see that, when the classical tachyonic velocity $u$ tends to infinity, i.e., when $\eta_{0} \rightarrow \infty$, then, the group velocity $v_{g}$ tends asymptotically to zero. In other words, in a reference frame moving with an infinitely large relative velocity, the wave packet is localized. This is another relevant result of this study and it allows to reinterpret the Hartman effect [27] in quantum tunnelling phenomena. This will be discussed in more detail in Section 5. Furthermore, the limit $v_{g} \rightarrow 0$ when $\eta_{0} \rightarrow \infty$ can be used to explain why the rest mass energy of the PT is given by $\mu c^{2}$. The imaginary value of this energy is due to the proper time $\tau$ of a tachyon which, as is known [28,29], is imaginary.

\section{Discussion}

In this study, we proved that, once appropriate initial conditions have been set, it is always possible to find a general solution of the TKG equation in which no superluminal components arise and that gives the same results of the ordinary wave equation at the limit $\left|\omega_{0}\right| \rightarrow 0$. Since each component of the tachyonic spinors must satisfy the TKG equation, we infer that all the possible tachyonic wave equations that can be formulated in the framework of quantum mechanics describe the dynamics of subluminal particles with spacelike momentum. This result has been validated for the Chodos equation. In this context, it is proved that the wave packet solution of this equation is characterized by a subluminal group velocity that tends asymptotically to zero as the corresponding classical particle velocity tends to infinitely large values. In other words, the tachyonic wave packet tends to behave like a localized wave for sufficiently small energy values. Such a behaviour could explain the long-standing controversy concerning the time spent by a massive particle to cross a classically forbidden barrier, i.e., the tunnelling time [30]. Theoretical 
investigations show that, for sufficiently large barriers, the scattering velocity may become superluminal [9]. However, recently, Ramos et al. have published an article [31] in which they state that, although a peak appears at the output before the input even arrives, it does not mean anything travelled faster than light. This is due to the fact that there is no law connecting an incoming and an outgoing peak. We believe that this law may have something to do with the fact that, within the potential barrier, the particle behaves like a nearly localized PT wave packet. Therefore, inside the barrier, the particle behaves like a bradyon which satisfies the spacelike energy-momentum relation. This suggests that the scattering theory of a generic massive particle impinging a classically forbidden potential barrier should be completely revised.

Funding: This research received no external funding.

Institutional Review Board Statement: Not applicable.

Informed Consent Statement: Not applicable.

Data Availability Statement: Not applicable.

Conflicts of Interest: The author declares that he has no known competing financial interests or personal relationships that could have appeared to influence the work reported in this paper.

\section{References}

1. Sommerfeld, A. Simplified deduction of the field and the forces of an electron, moving in a given way. Proc. Amst. Acad. 1904, 8 , 346-367.

2. Heaviside, O. Electrical Papers; Macmillian: London, UK, 1892; Volume II.

3. Nahin, P.J. Oliver Heaviside: An accidental time traveller. Phil. Trans. R. Soc. A 2018, 376. [CrossRef]

4. Bilaniuk, O.M.P.; Dashpande, V.K.; Surdarshan, E.C.G. Meta Relativity. Am. J. Phys. 1962, 30, 718-723. [CrossRef]

5. Bilaniuk, O.M.P.; Surdarshan, E.C.G. Particles beyond the Light Barrier. Phys. Today 1968, 22, 43-51. [CrossRef]

6. Feinberg, G. Possibility of Faster-Than-Light Particles. Phys. Rev. 1967, 159, 1089-1105. [CrossRef]

7. Ecker, G. Quantum Field Theory with Space-Like Momentum Spectrum. Ann. Phys. 1970, 58, 303-321. [CrossRef]

8. Ehrlich, R. Faster-Than-Light Speeds, Tachyons, and the Possibility of Tachyonic Neutrinos. Am. J. Phys. 2003, 71, 1109-1114. [CrossRef]

9. Recami, E. Superluminal Waves and Objects: An Overview on the Relevant Experiments. J. Phys. Conf. Ser. 2009, 196, 012020. [CrossRef]

10. Recami, E. Tachyon Kinematics and Causality: A Systematic Thorough Analysis of the Tachyon Causal Paradoxes. Found. Phys. 1987, 17, 239-296. [CrossRef]

11. Bose, S.K. Aspects of Tachyon Theory. J. Phys. Conf. Ser. 2009, 196, 012022. [CrossRef]

12. Adamson, P.; Andreopoulos, C.; Arms, K.E.; Armstrong, R.; Auty, D.J.; Avvakumov, S.; Ayres, D.S.; Baller, B.; Barish, B.; Barnes, P.D., Jr.; et al. Measurement of Neutrino Velocity with the MINOS Detectors and NuMI Neutrino Beam. Phys. Rev. D 2007, 76, 072005. [CrossRef]

13. Mohseni Sadjadi, H.; Anari, V. Mass Varying Neutrinos, Symmetry Breaking, and Cosmic Acceleration. Phys. Rev. D 2017, 95, 123521. [CrossRef]

14. Backenstoss, G.; Daniel, H.; Koch, H.; Von der Malsburg, C.; Poelz, G.; Povel, H.P.; Schmitt, H.; Tauscher, L. $\pi^{-}$mass and vacuum polarization. A re-evaluation. Phys. Lett. B 1973, 43, 539-541. [CrossRef]

15. Lu, D.C.; Delker, L.; Dugan, G.; Wu, C.S.; Caffrey, A.J.; Cheng, Y.T.; Lee, Y.K. New High Accuracy Measurement of the Pionic Mass. Phys. Rev. Lett. 1980, 45, 1066-1069. [CrossRef]

16. Soucek, J. Quaternion Quantum Mechanics and the Description of Tachyons and Quarks. Czechoslov. J. Phys. B 1979, 29, 315-318. [CrossRef]

17. Schwartz, C. Toward a Quantum Theory of Tachyon Fields. Int. J. Mod. Phys. A 2016, 31, 1650041. [CrossRef]

18. Salesi, G. Slower-Than-Light Spin-1/2 Particles Endowed with Negative Mass Squared. Int. J. Mod. Phys. A 1985, 28, 5103. [CrossRef]

19. Jentschura, U.D.; Wundt, B.J. Pseudo-Hermitian Quantum Dynamics of Tachyonic Spin-1/2 Particles. J. Phys. A Math. Theor. 2012, 45, 444017. [CrossRef]

20. Chodos, A.; Hauser, A.I. The Neutrino as a Tachyon. Phys. Lett. B 1985, 150, 295-302. [CrossRef]

21. Partington, J.R. Fourier Transforms and Complex Analysis. In Harmonic Analysis and Rational Approximation; Fournier, J.D., Grimm, J., Leblond, J.R., Partington, J.R., Eds.; Lecture Notes in Control and Information Science; Springer: Berlin/Heidelberg, Germany, 2006; Volume 327.

22. Kammler, D.W. A First Course in Fourier Analysis, 2nd ed.; Cambridge University Press: Cambridge, UK, 2008. 
23. Hecht, K.T. The Motion of Wave Packets: Fourier Analysis. In Quantum Mechanics; Graduate Texts in Contemporary Physics; Springer: New York, NY, USA, 2000.

24. Jentschura, U.D. Tachyonic Field Theory and Neutrino Mass Running. Cent. Eur. J. Phys. 2012, 10, 749-762. [CrossRef]

25. Tanaka, S. Theory of Matter with Super Light Velocity. Prog. Theor. Phys. 1960, 24, 177-181. [CrossRef]

26. Park, S.T. Propagation of a Relativistic Electron Wave Packet in the Dirac Equation. Phys. Rev. A 2012, 86, 062105. [CrossRef]

27. Winful, H.G. Tunneling time, the Hartman effect, and superluminality: A proposed resolution of an old paradox. Phys. Rep. 2006, 436, 1-69. [CrossRef]

28. Recami, E.; Mignani, R. Classical theory of tachyons (special relativity extended to superluminal frames and objects). Riv. Nuovo Cim. 1974, 4, 209-290. [CrossRef]

29. Dawe, R.L.; Hines, K.C. The Physics of Tachyons. Aust. J. Phys. 1992, 45, 591-620. [CrossRef]

30. Davies, P.C.W. Quantum Tunnelling Time. Am. J. Phys. 2005, 73, 23-27. [CrossRef]

31. Ramos, R.; Spierings, D.; Racicot, I. Steinberg, A.M. Measurement of the time spent by a tunnelling atom within the barrier region. Nature 2020, 583, 529-532. [CrossRef] 